Research Article

\title{
Research and Implementation of SVDU Simulator Based on Emulation Technology
}

\author{
Yanqun Wu $\mathbb{D}^{1},{ }^{1}$ Mingxing Liu $\mathbb{D},{ }^{1,2}$ Qi Chen, ${ }^{1}$ Xu Zhang, ${ }^{1}$ Xufeng Tian, ${ }^{1}$ Yu Zhang, \\ and Meiyuan Chen ${ }^{1}$ \\ ${ }^{1}$ Science and Technology on Reactor System Design Technology Laboratory Nuclear Power Institute of China, \\ Chengdu 610213, China \\ ${ }^{2}$ School of Computer Science, Sichuan University, Chengdu 610065, China
}

Correspondence should be addressed to Yanqun Wu; 15720123548@163.com

Received 11 January 2021; Accepted 31 May 2021; Published 12 June 2021

Academic Editor: Massimo Zucchetti

Copyright (c) 2021 Yanqun Wu et al. This is an open access article distributed under the Creative Commons Attribution License, which permits unrestricted use, distribution, and reproduction in any medium, provided the original work is properly cited.

The safety video display unit (SVDU), as the display machine of the reactor protection system, performs the functions of displaying the reactor's safety parameters and sending safety control commands. In order to meet the needs of nuclear power safety-level digital control system (DCS), like designing verification, operator training, and accidental drills, for the SVDU in the NASPIC platform developed independently by China National Nuclear Corporation, a virtual embedded system technology based on the micro x86 industrial host is proposed to make software simulation to the SVDU physical controller with exactly the same hardware appearance of the original one. The SVDU stimulator realized by this research can achieve $100 \%$ simulation of the logical functions of the SVDU physical equipment and the synchronized upgrading function between stimulator and the real equipment. With the development of multiple engineering application requirements, such as configuration verification and operator training, this stimulator has been applied in several virtual security level DCS projects.

\section{Introduction}

Nuclear power plant (NPP) safety-level instrumentation and control (I\&C) systems have gone through two stages: instrument control systems and semidigital control systems and have fully turned to the era of DCS. Accompanied with the upgrading of the technology of I\&C systems used in NPP, the simulation technology especially for I\&C systems develops as well. Multiple technologies grow generally from the original pure simulation, like simulation, emulation, translate simulation, and physical stimulation [1]. Considering the complicated structure and diversity functions, the old pure simulation and translate simulation cannot match the latest requirements of high repetition, rapid verification, operator training, accidental drills, or design optimization in comprehensive and integrated aspects. The emulation technology which uses simple computing system to replace the complex physical hardware system simulates the embedded hardware operating environment through computers [2], applies the operating software and configuration data exactly the same in the real one, and can reproduce the hardware, software, and control function features, with higher realistic and lower cost on operation and maintenance, which can meet the multidimensional simulation application requirements of the I\&C system.

The simulation of the I\&C system is widely researched and applied in various industries. On the basis of the full scope simulator, L. Daogang et al. used the RELAP5/MOD 3.4 code to model the secondary circuit and realized the realtime full scope simulation under the transient power changes and accident scenarios [3]. In parallel with the design and construction of the Keystone pipeline, TransCanada and Telvent developed a fully integrated simulation environment including a dynamic hydraulic model [4]. Kozlováet al. used the data collected at control room full scope simulator of Dukovany Nuclear Power Plant to analyze human factor reliability [5]. Golsa et al. proposed a vehicular channel emulator architecture which aims at a 
hardware implementation which requires minimal hardware complexity for emulating channels of safety-relevant vehicular scenarios [6]. Youssef Mallal et al. presented the design and implementation of a photovoltaic emulator to research the characteristics of photovoltaic panels, and the emulator was able to behave accurately as the studied photovoltaic panel [7].

There are also many studies on simulators in the field of nuclear safety-level DCS. Studying the NASPIC system of CNNC, Feifei et al. designed a high accuracy virtual DCS platform based on virtual slave computer technology [8]. By analyzing the characteristics of CEPR nuclear power technology, Peijin et al. proposed a DCS simulation mode combining virtual simulation and functional simulation [1] and Weichang et al. proposed a virtual simulation method which firstly establishes the control logic model before the virtual simulation [2]. Hanjun et al. put forward the method of using virtual DCS controller instead of physical controller during the DCS prototype logic tests [9].

As an important part of safety-level DCS, SVDU provides operators with a direct interactive tool to understand and control the operation status of the reactor. The accuracy of its simulation is the key to the design of safety-level DCS simulator. Most of the safety-level DCS simulators emulate SVDU less or only adopt simulation or translation simulation, while few are focused on SVDU high accuracy simulation and the safety characteristics of SVDU $[10,11]$. In this paper, a virtual embedded system technology is proposed to simulate the SVDU physical controller based on the micro $x 86$ industrial host. The hardware and structure of the SVDU are designed $1: 1$ to achieve the best simulation performance between the SVDU simulator and the real machine. The simulation functions such as stepping, running, freezing, fast and slow speed, working condition, and environment are realized to meet the requirements of configuration verification, operator training, accidental drills, simulation test, and other engineering requirements.

\section{SVDU of NASPIC}

NASPIC is a safety-level distributed control system independently developed by CNNC. It is composed of field control station, transmission unit, safety video display unit, gateway station, and maintenance test station. The relationship between the SVDU and other components of NASPIC platform is shown in Figure 1.

SVDU is an integrated structure with ARM and FPGA as its main processors, which can be installed in the panel or field cabinet of the main control room. The integrated machine form of SVDU is shown in Figure 2.

\section{Functional Requirements of SVDU Simulator}

SVDU simulator is a part of the safety-level DCS simulation system. It not only can cooperate with the safety-level DCS simulation system to realize the full scope of simulator functions but also can be independently applied to other business scenarios such as functional preresearch and digital twin. In the business scenario of nuclear power full scope simulator, SVDU simulator and safety-level DCS simulation system have interface relationship with process model, I\&C instructor station, and nonsafety-level DCS in full scope simulator. The interface relationship between SVDU simulator and other parts in the full scope simulator is shown in Figure 3.

The SVDU simulator needs to simulate all the core functions of the SVDU physical equipment, with simulation interaction, data management capabilities, and the corresponding simulation management functions to meet the needs of engineering applications at the mean time. In order to meet the above requirements, the SVDU simulator needs to implement the following:

(i) Almost $100 \%$ is approached in the virtual reality. SVDU simulation must not only achieve a high degree of approximation of appearance and structure but also complete the simulation of the logical functions and human-computer interaction functions of the SVDU physical equipment.

(ii) Fully compatible with the SVDU configuration file of the actual DCS, and achieve the same configuration screen and control functions as the actual SVDU.

(iii) The SVDU simulator needs to realize rich simulation management functions, including start, stop, step, run, freeze, fast, slow, snap, backtracking, replay, scene simulation, over control, and fault simulation.

(iv) There were no less than 5000 hours of historical data depth. The SVDU simulator needs to support 100 scene files storage, and each scene file can save 50 hours of uninterrupted running data.

\section{Design of SVDU Simulator}

4.1. Overall Design. The SVDU simulator is mainly composed of a micro x86 industrial host, an integrated antivibration structure, hardware peripherals, configuration simulator, and simulation manager, which is shown in Figure 4 .

4.2. Hardware and Structure Design. The microindustrial host with small dimension, fast calculation speed, and excellent expansibility is applied to realize the logic processing and data communication functions of ARM and FPGA and to meet the calculation requirements like huge capacity data reading and writing or simulation management function scheduling.

The hardware peripherals provide power supply, data display, communication, command input, and other functions. An important one is the expansion on huge volume solid-state hard disk, which is used to support the numerous data created during the simulation process. The overall hardware design block diagram of the SVDU simulator is shown in Figure 5, which is mainly composed of microindustrial host, power filter, I/O signal transfer board, driver board, touch screen, and LCD. 


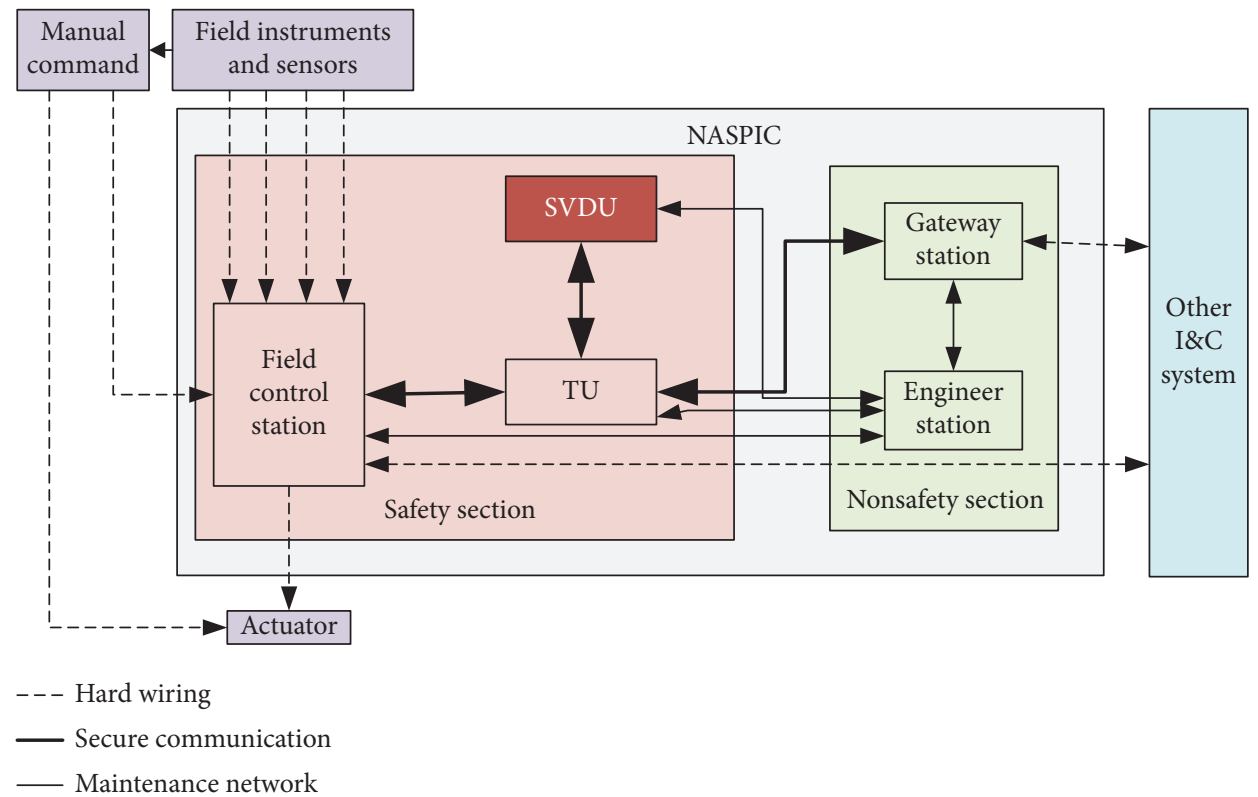

FIgURE 1: Composition relationship between SVDU and NASPIC platform.

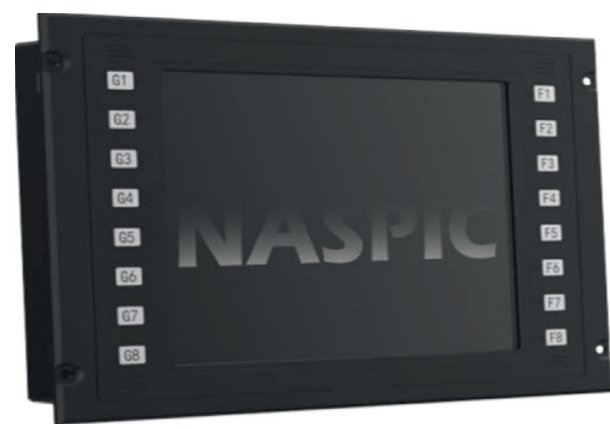

FIGURE 2: The integrated machine form of SVDU.

The simulator uses the exactly same LCD screen, key panel, and resistive touchscreen as the real SVDU's to rebuild the appearance and operation type of the real one. The all-in-one structure is used on the simulator to reduce the interface between the separate components and to further decrease the fault points to increase the stability. The structure of the SVDU simulator is divided into two parts: the main body and the back cover. The three-dimensional assembly diagram is shown in Figure 6. The screen components and keys are fixed in the cavity of the main body, and the micro $\mathrm{x} 86$ host and power module are fixed on the back cover plate.

\subsection{Software Design}

4.3.1. Software Framework. The SVDU simulator software is mainly composed of two parts: the configuration simulator and the simulation manager, as shown in Figure 7. The configuration simulator realizes the simulation of the SVDU core functions, and the simulation manager mainly realizes the functions of communication, scheduling, simulation management, and data management.
4.3.2. Configuration Simulator. The configuration simulator uses the virtual embedded system technology, porting the embedded code running on the SVDU entity device to the micro x86 platform, realizes the analysis and simulation operation of the configuration data generated by the configuration compiler, and be able to record the running process data for a long time. The overall architecture of the SVDU configuration simulator is shown in Figure 8.

The configuration simulator can run in simulation running stage and replay running stage. The instruction control module transforms the control scheduling instruction generated by the simulation manager into corresponding simulation instruction and realizes simulation in different stages. The configuration simulator and the physical machine have the same logical behavior and time characteristics during the simulation process. The difference is that the memory variables and man-machine inputs periodically generated by the configuration parser in the SVDU simulator are sent to the output buffer and stored, while these data will not be stored in the SVDU physical device. In the replay running stage, the configuration parser loads the stored man-machine input, memory variables, and other data stored in the simulation running stage and performs the corresponding operation and display to realize the replay of the finished process.

\subsubsection{Simulation Manager}

(1) Main Scheduling Service. As the top layer of SVDU simulator software structure, the main scheduling service realizes the functions as follows:

(i) The operation control of each module and the data exchange between modules

(ii) Receiving the instruction data of communication module and reacting according to the instruction 


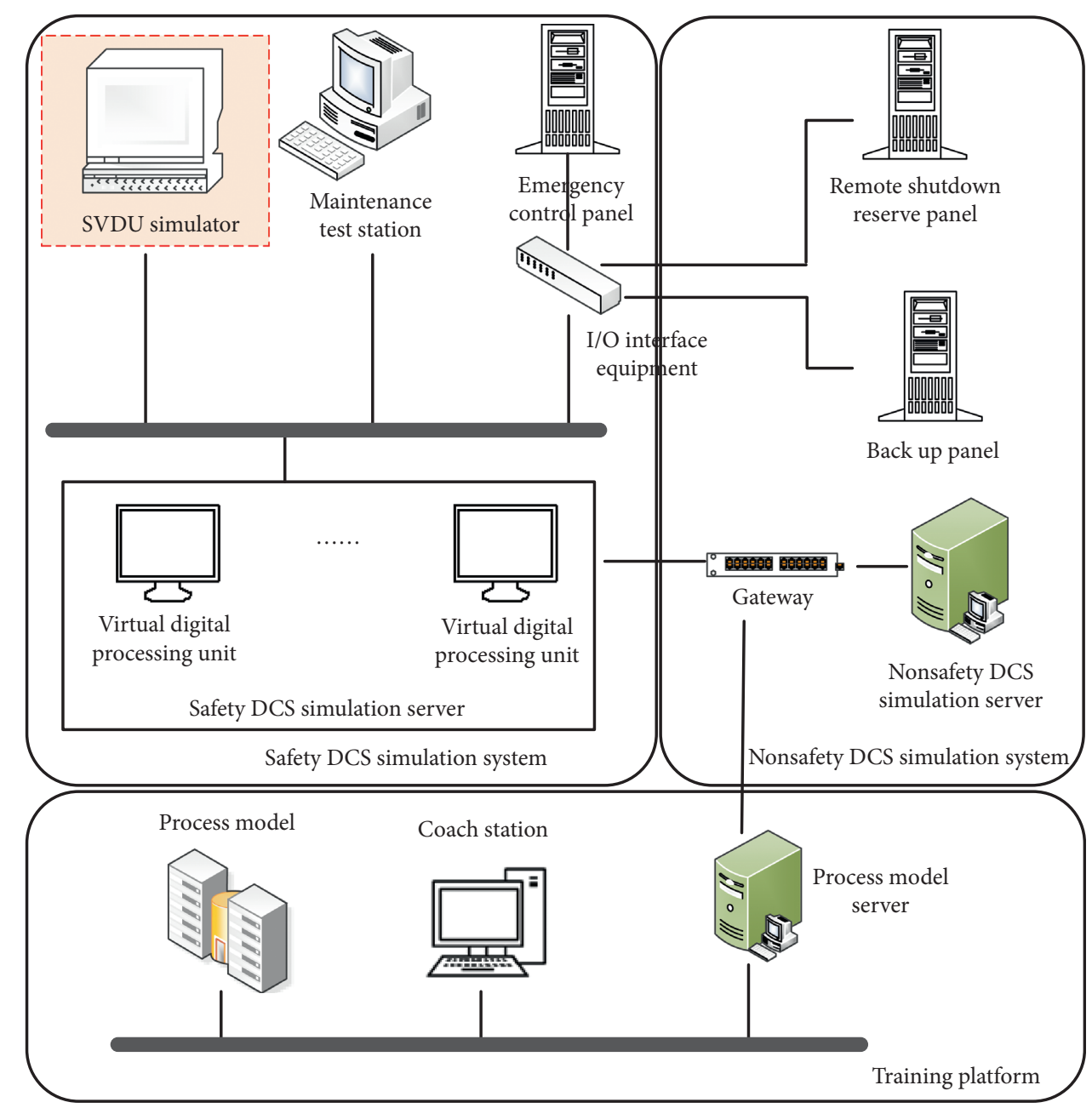

FIGURE 3: Interface relationship between SVDU simulator and other parts in full scope simulator.

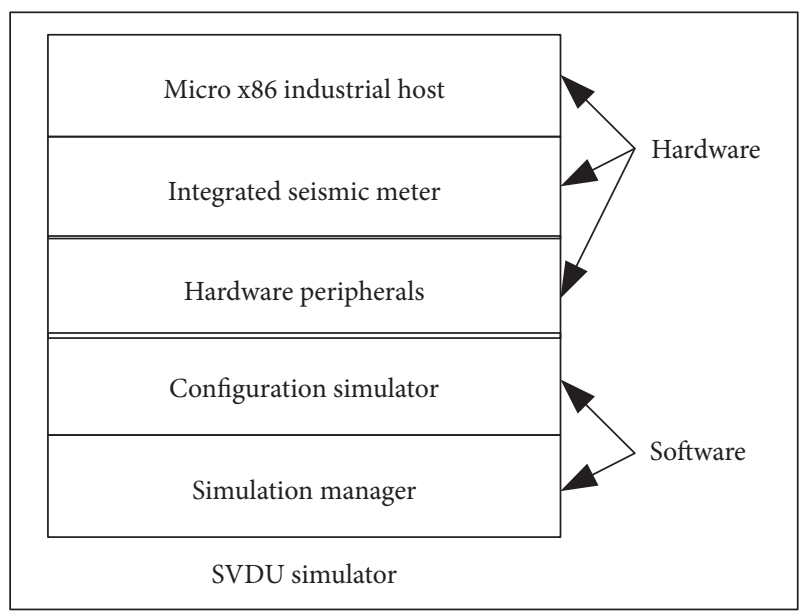

FIgURE 4: Overall design of the SVDU simulator.

(iii) Encapsulating SVDU kernel data and reducing the contact between the kernel and other modules

(2) Simulation Instruction Control Module. The SVDU simulator has many simulation instructions, and the state transition between the instructions is complicated. The simulation instruction control module is responsible for the state switching and controlling of the SVDU simulation process to ensure accurate state switching of the simulation process. The design of the simulation process is shown in Figure 9. After the simulator is powered on, it waits for the simulation instruction to start and enters different simulation states according to the latest setting mode.

(3) Communication Module. The communication module is responsible for receiving the network packets, grouping the received network data according to the simulation instruction data format, and sending the packet data to the scheduling service. In addition, the communication module provides communication services as the download interface of data files, configuration files, and engineering files communicating with MTS. The link between the SVDU simulator and the MTS is realized through a switch, and TCP/IP protocol is used as the communication protocol, as shown in Figure 10.

(4) Storage Design. SVDU is a human-machine interaction device that requires a large amount of internal variable 


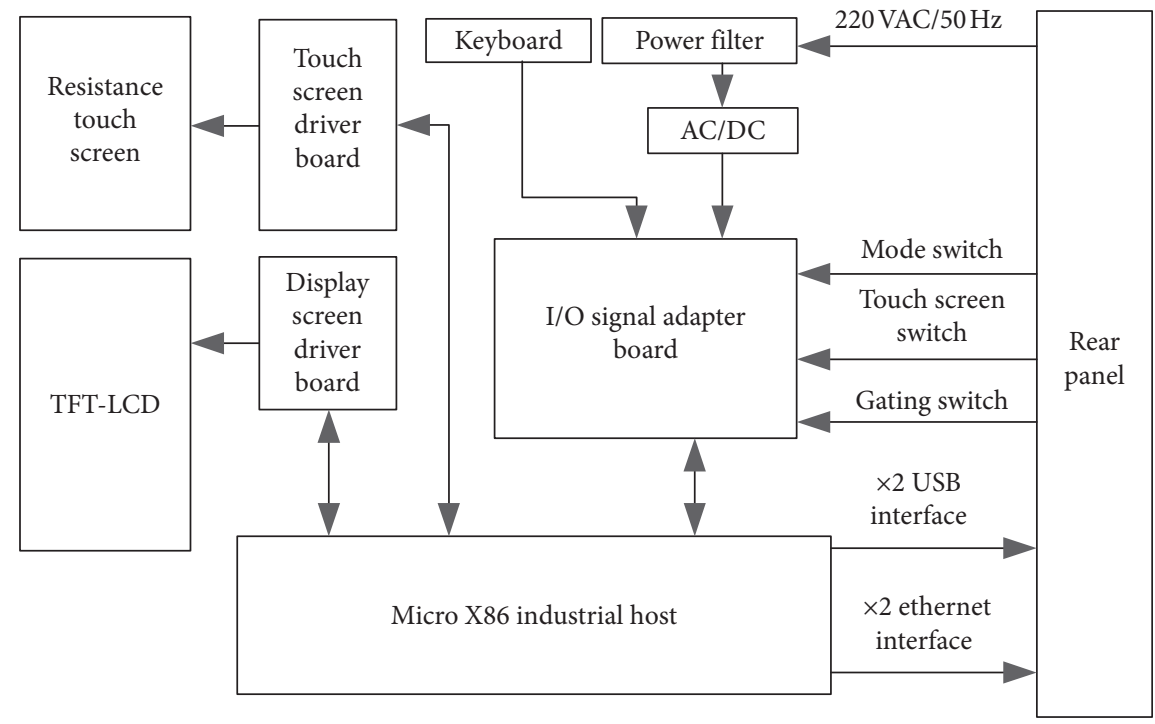

FIGURE 5: Overall hardware design of the SVDU simulator.

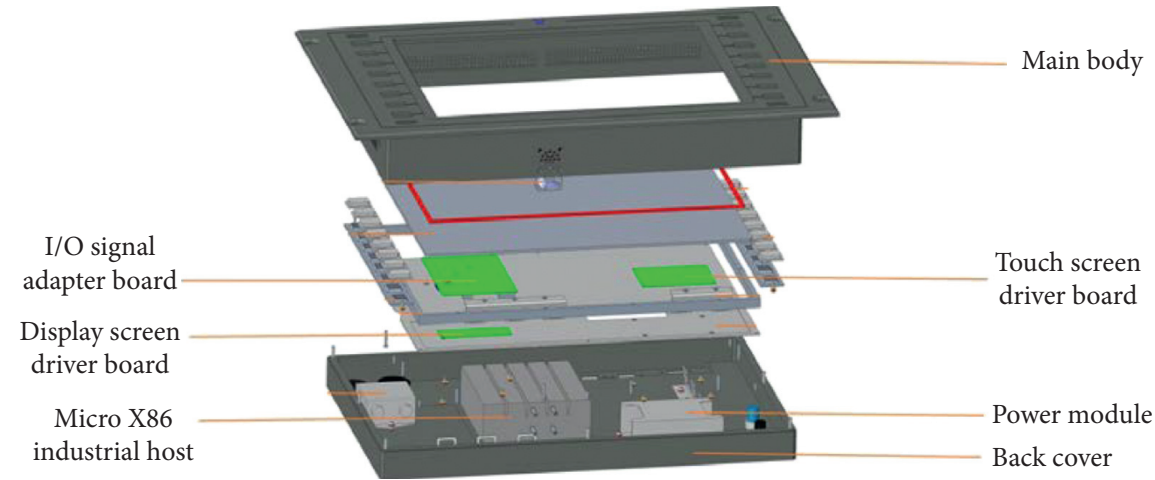

FIGURE 6: Three-dimensional assembly drawing of the simulator.

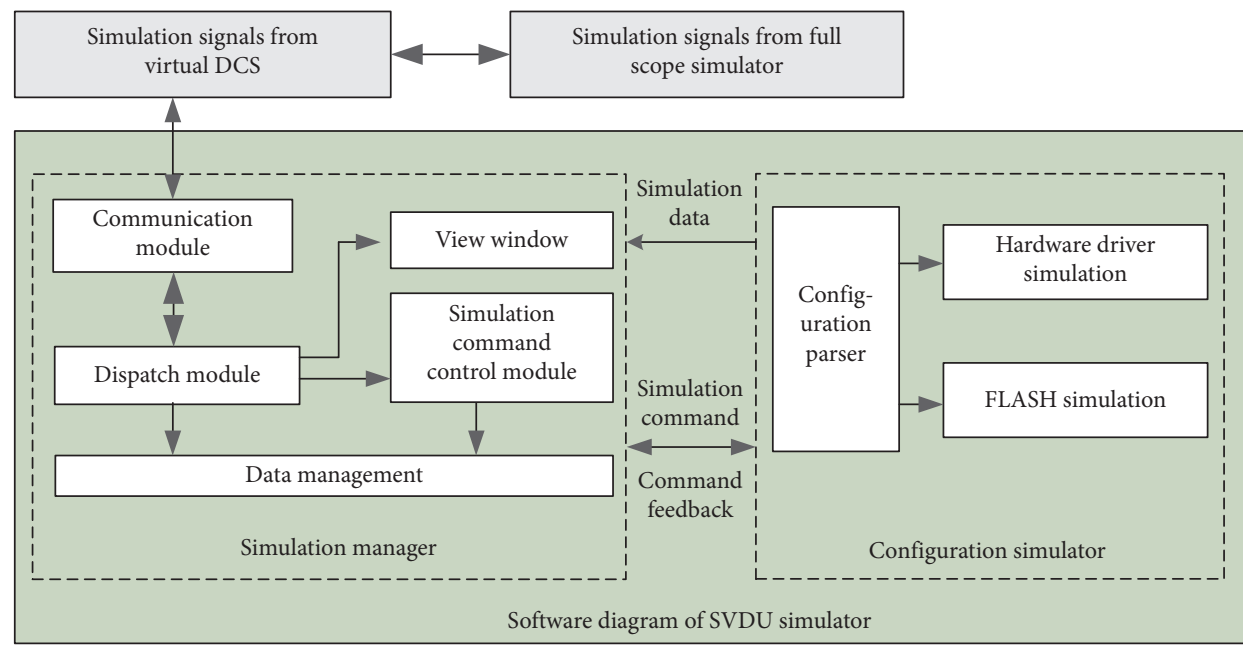

FIgURE 7: Software block diagram of the SVDU simulator.

information to support the correct display and interaction of the graphic unit during running. The data that the SVDU simulator need to manage and store can be divided into human-machine operation data, configuration screen data, and internal variable data according to the data function type. Without a data server, the 


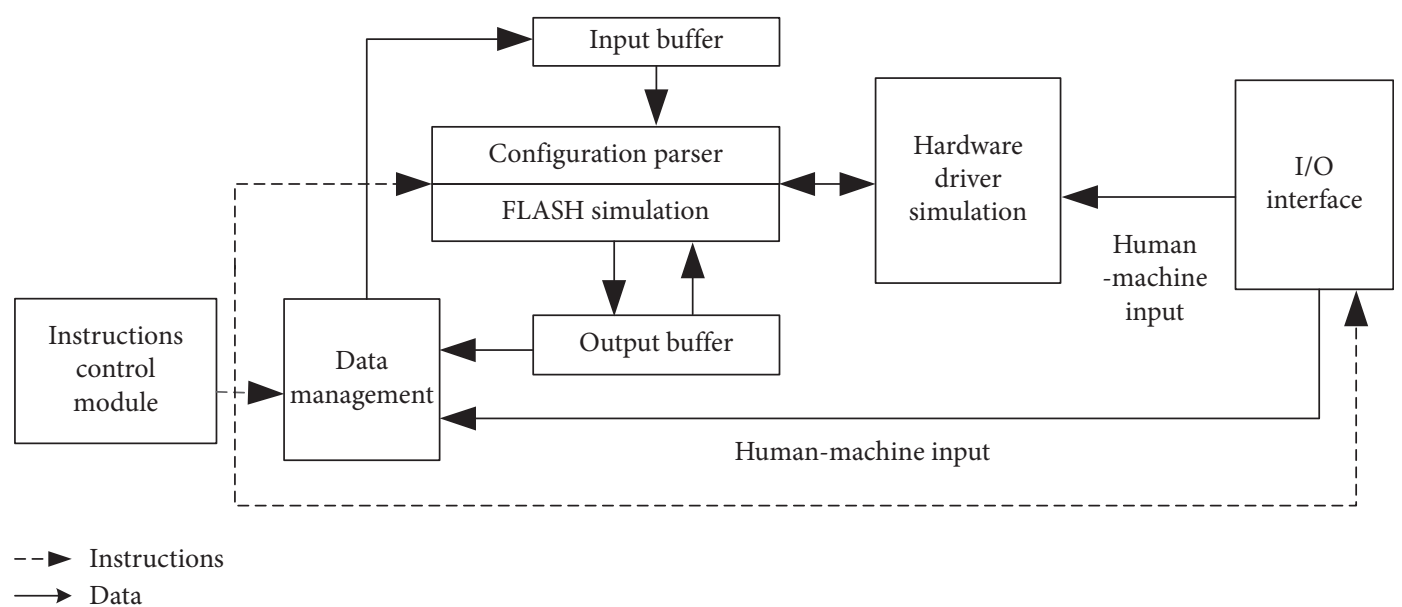

FIgURE 8: Overall architecture of the SVDU configuration simulator.

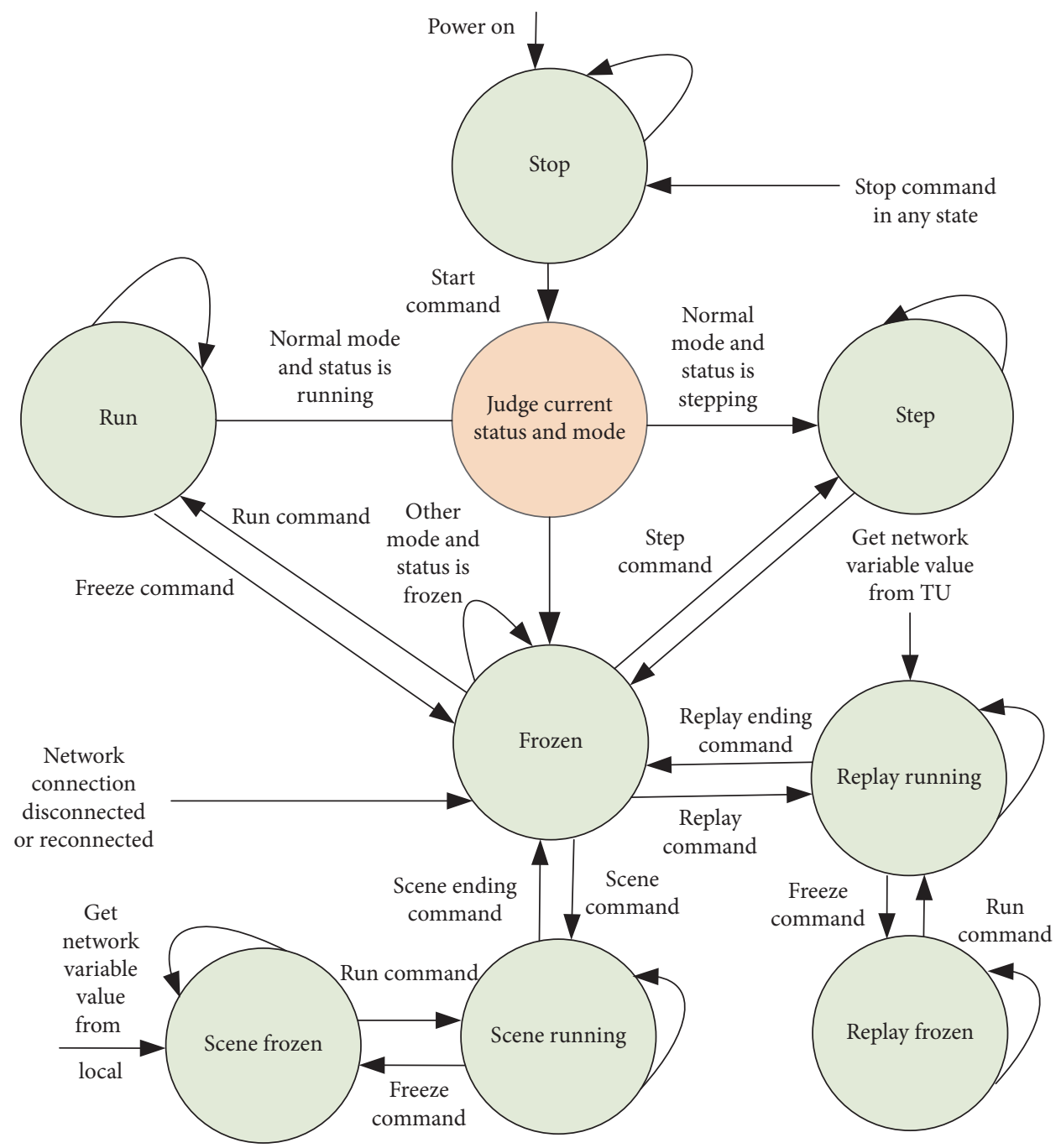

FIGURE 9: State switching diagram of simulation process.

SVDU simulator itself needs to store 5000 hours of process and historical data.

In order to reduce the data volume, on the premise of meeting the functional requirements, a data differential storage method is figured out. This method uses 15 minutes long data piece to be managed by the SVDU simulator as a data block. Each data block contains collection timestamps, internal variable basic data, per-minute change data, 1- 


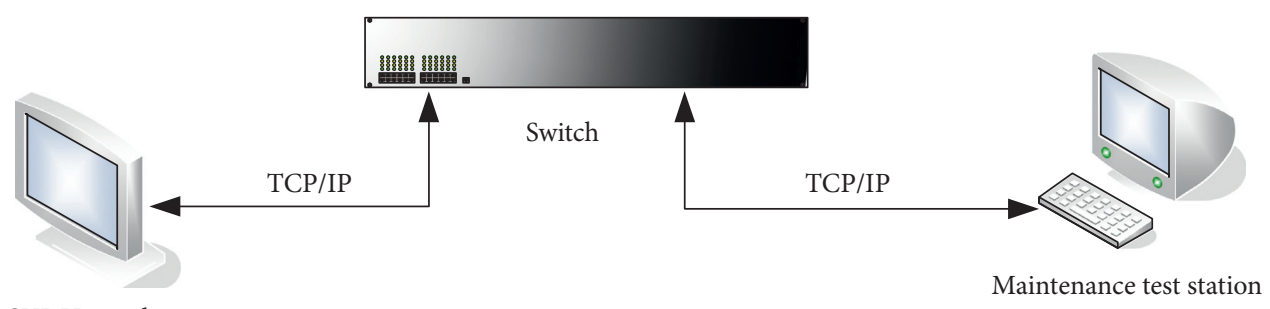

SVDU simulator

FIGURE 10: Schematic diagram of connection between SVDU simulator and maintenance test station.

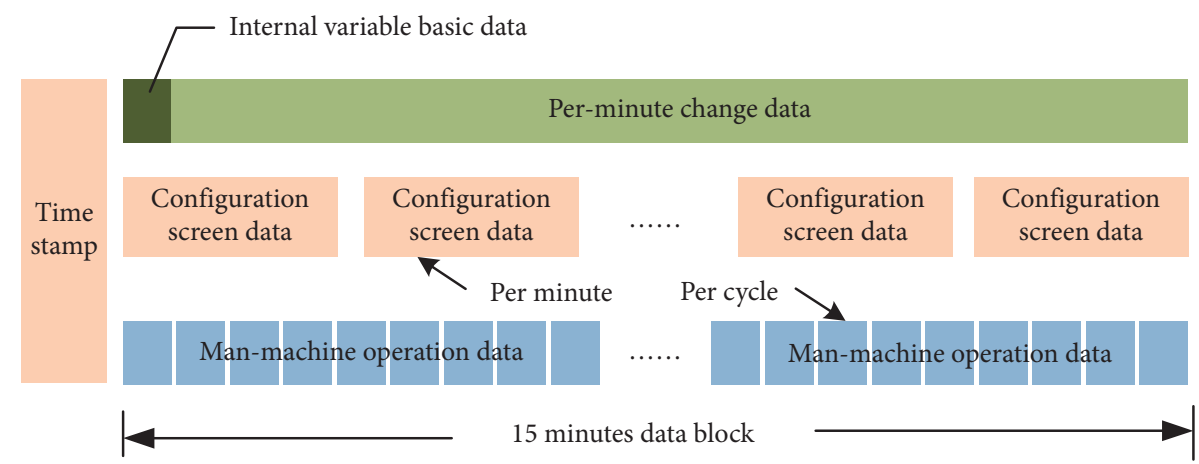

FIGURE 11: Data structure of the SVDU simulator.

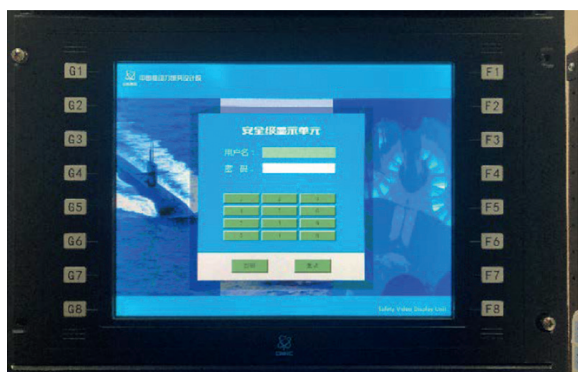

(a)

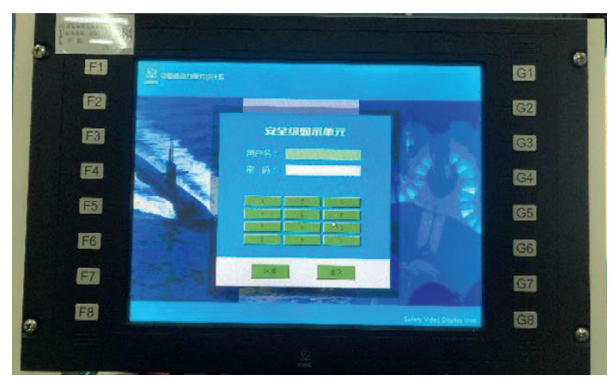

(b)

Figure 12: Physical picture: (a) SVDU physical equipment; (b) SVDU simulator.

minute cycle screen data, and man-machine operation data within 15 minutes. The data structure is shown in Figure 11.

\section{Implementation of SVDU Simulator}

The appearance and structure of the SVDU simulator based on the above design are almost the same as those of the SVDU physical equipment. The actual appearance is shown in Figure 12.

The SVDU simulator can directly run the DCS configuration file downloaded from the maintenance test station. It can work in the independent simulation mode and joint simulation mode. When working in the independent simulation mode, it generates simulation instructions by itself to perform corresponding simulation functions; when working in cosimulation mode, it receives simulation instructions from the full scope simulators. After testing and engineering application verification, the performance of the SVDU simulator meets the requirements of Table 1.
According to tests and verification, the SVDU simulator can fully simulate all functions of SVDU physical equipment, such as data display, control command transmitting, mode switching, download, and the simulation management function runs accurately, meeting the engineering application requirements of configuration verification, operator training, accidental drills, simulation test, and so on.

FangJiaShan Full Scope Simulator System and TianWan Unit 5 and 6 Full Scope Simulator System are widely used simulator systems for nuclear power plant operator training. This paper selects the SVDU simulator of the two and the SVDU simulator developed in this paper for some functional performance comparisons. The comparison results are shown in Table 2.

The SVDU simulator based on NASPIC can not only reproduce the software and control features of SVDU but also has good performance in simulation control and 
TABLE 1: Performance parameters of the SVDU simulator.

\begin{tabular}{lc}
\hline Parameter content & Parameter value \\
\hline Boot time & $<1 \mathrm{~min}$ \\
Shut down time & $<1 \mathrm{~min}$ \\
Loading condition time & $<30 \mathrm{~s}$ \\
Loading backtracking condition time & $<30 \mathrm{~s}$ \\
Max number of storage conditions & 1000 \\
Max number of backtracking conditions & 60 \\
Max number of scene files & 100 \\
Max support on fast/slow speed & 2 times/10 times \\
Data depth of each scene file & $50 \mathrm{~h}$ \\
Time error & $<5 \mathrm{~ms}$ \\
Data refresh period & $<200 \mathrm{~ms}$ \\
Communication speed & $>100 \mathrm{Mbps}$ \\
\hline
\end{tabular}

TABLE 2: Comparison results of SVDU simulators.

\begin{tabular}{|c|c|c|c|}
\hline Compare items & $\begin{array}{l}\text { SVDU simulator of } \\
\text { NASPIC }\end{array}$ & $\begin{array}{l}\text { SVDU simulator of } \\
\text { FangJiaShan }\end{array}$ & $\begin{array}{l}\text { SVDU simulator of } \\
\text { TianWan }\end{array}$ \\
\hline Simulation type & Emulation & Translation simulation & Translation simulation \\
\hline Fidelity & $\star \star \star$ & $\star$ & $\star$ \\
\hline Software and control features & $\star \star \star$ & $\star \star$ & $\star \star$ \\
\hline Simulation of hardware appearance & $\star \star \star$ & ts & ts \\
\hline $\begin{array}{l}\text { Simulation of hardware control type and } \\
\text { touch }\end{array}$ & $\star \star \star$ & is & is \\
\hline $\begin{array}{l}\text { Simulation of software security } \\
\text { characteristics }\end{array}$ & $\star \star \star$ & is & is \\
\hline $\begin{array}{l}\text { Functional requirements of simulation } \\
\text { control }\end{array}$ & $\star \star \star$ & $\star \star \star$ & $\star \star \star$ \\
\hline Simulation performance requirements & $\star \star \star$ & $\star \star \star$ & $\star \star \star$ \\
\hline
\end{tabular}

$\star$ represents the degree of fidelity with the real SVDU such as $\star \star \star$ : high; $\star \star$ : medium; $\star$ : low; ; : null.

performance. Moreover, it is consistent with the real SVDU in hardware appearance simulation, hardware touch, and software security performance.

\section{Conclusion}

In this paper, the functional requirements of the SVDU in the full scope simulator of safety-level DCS are analyzed, and the SVDU simulator is studied from the aspects of overall, hardware, structure, and software design. According to these studies, the design scheme of the SVDU simulator based on micro x86 industrial host and virtual embedded system technology is proposed and implemented. At present, the SVDU simulator has been applied in the FT test of NASPIC platform and has been applied in a full scope simulator system of a certain reactor. As a kind of simulation equipment, the SVDU simulator can be used as a part of the full scope simulator to perform the simulation function. It can also be used as a software study platform for the preresearch of new functions. Besides, it can be further improved into a graphic interface for the nonsafety part of safety-level DCS, which has a bright future of function preresearch and engineering application.

\section{Data Availability}

The data used in this paper belong to the intellectual property rights of the laboratory, which is proprietary and cannot be disclosed.

\section{Conflicts of Interest}

The authors declare that they have no conflicts of interest.

\section{Acknowledgments}

This work was supported by the Sichuan Science and Technology Fund for Outstanding Youth (project no. 2020JDJQ0068).

\section{References}

[1] L. Peijin, Z. Weichang, X. Liangjun, and Z. Liqiang, "Optimization analysis of synthesized FSS simulation method applied for DCS design evolution," Nuclear Power Engineering, vol. 36, no. S1, pp. 9-13, 2015.

[2] Z. Weichang, B. Xiushi, D. Jijie, and C. Hao, "Key techniques of CEPR full scope simulator," Nuclear Power Engineering, vol. 36, no. S1, pp. 67-70, 2015.

[3] D. G. Lu, F. Zhang, D. T. Sui et al., "Full scope modeling and analysis on the secondary circuit of Chinese large-capacity advanced PWR based on RELAP5 code," Science \& Technology of Nuclear Installations, vol. 2015, no. 13, pp. 479-486, 2016.

[4] K. Nelson, S. Smith, and M. Malinowski, "Developing and Implementing a "Full Scope" Operator Trainer Simulator for the TransCanada Keystone Pipeline," in Proceedings of the PSIG Annual Meeting, Bonita Springs, FL, USA, May 2010.

[5] K. Kozlová, K. Barčová, and J. Kubíček, "Analysis of data collected at control room full scope simulator at Dukovany nuclear power plant," Transactions of the Vb-Technical 
University of Ostrava Safety Engineering, vol. 12, no. 2, pp. 11-22, 2018.

[6] G. Golsa, B. Thomas, A. Mehdi et al., "Real-time emulation of nonstationary channels in safety-relevant vehicular scenarios," Wireless Communications \& Mobile Computing, vol. 2018, Article ID 2423837, 11 pages, 2018.

[7] Y. Mallal, L. El Bahir, and T. Hassboun, "High-performance emulator for fixed photovoltaic panels," International Journal of Photoenergy, vol. 2019, Article ID 3951841, 11 pages, 2019.

[8] Z. Feifei, X. Haiyan, Z. Yu, L. Mengshu, C. Meiyuan, and Y. Hao, "Research and implementation of virtual DCS based on NASPIC," Journal of Shanghai Jiaotong University, vol. 52, no. S1, pp. 133-136, 2018.

[9] G. Hanjun, L. Peibang, and L. Xingyan, "The applied DCS virtual machine in DCS prototype of NPP," Science \& Technology Vision, vol. 2, p. 258+250, 2017.

[10] W. Youguang, L. Mingming, Z. Zipeng, and L. Qian, "Research on technical scheme of safety level DCS simulation verification platform," Nuclear Power Engineering, vol. 41, no. 1, pp. 89-92, 2020.

[11] G. Hanjun, Q. Ming, Li Qin, and J. Yinggang, "Research and design on virtual DCS process control platform in nuclear power plant," Computer Simulation, vol. 34, no. 2, pp. 144149, 2017. 